\title{
Impact of endoscopic ultrasound on diagnosis and management of presumed mucinous neoplasms when done for pancreatic cyst morphology change on non-invasive surveillance imaging
}

\section{(우웅 $\odot$}

\author{
Authors \\ Ronald Salem ${ }^{3,4}$, James J Farrell ${ }^{2,3}$ \\ Institutions \\ 1 Department of Internal Medicine, Yale School of \\ Medicine, New Haven, CT \\ 2 Department of Digestive Diseases, Yale School of \\ Medicine, New Haven, CT \\ 3 Yale Center for Pancreatic Disease, Yale School of \\ Medicine, New Haven, CT \\ 4 Department of Surgical Oncology, Yale School of \\ Medicine, New Haven, CT
}

Kamraan Madhani ${ }^{1}$, Muhammad Yousaf ${ }^{2,3}$, Ali Aamar ${ }^{1}$, Kohtaro Ooka ${ }^{1}$, Thiruvengadam Muniraj ${ }^{2,3}$, Harry Aslanian²,3,

submitted 11.1.2018

accepted after revision $\quad 6.8 .2018$

Bibliography

DOI https://doi.org/10.1055/a-0824-6240 |

Endoscopy International Open 2019; 07: E389-E395

(c) Georg Thieme Verlag KG Stuttgart · New York

ISSN 2364-3722

Corresponding author

James J. Farrell, MD, Section of Digestive Diseases, Yale School of Medicine, LMP 1080, 15 York St, New Haven,

CT 06510-3221

Fax: +1-203-737-1755

james.j.farrell@yale.edu

\section{ABSTRACT}

Background and study aims Guidelines for management of presumed neoplastic pancreatic cysts have encouraged noninvasive imaging for low-risk surveillance, while reser- ving endoscopic ultrasound for worrisome features including morphologic change. We aim to study the impact of endoscopic ultrasound on diagnosis and management compared with non-invasive imaging.

Patients and methods A single-institution pancreatic cyst database was retrospectively queried for patients who underwent endoscopic ultrasound for the indication of change in cyst morphology. Diagnoses were classified as presumed mucinous neoplasm with or without worrisome features or high-risk stigmata and non-mucinous lesions. Management decisions were defined a priori as surgical evaluation for patients with high-risk stigmata, positive cytology or mural nodule, or continued surveillance for all others.

Results Between January 2013 and October 2016, 709 pancreas cyst endoscopic ultrasounds were performed of which 89 were for cyst morphology change seen on noninvasive imaging including 10 presumed pseudocysts, nine presumed serous cystadenomas, and 70 presumed mucinous cystic neoplasms. Cyst morphologic changes included increase in caliber of the main pancreatic duct (7 cases), increase in cyst size (68 cases), cyst $\geq 30 \mathrm{~mm}$ (10 cases), and presence of a solid nodule ( 1 case). Median cyst size increase was $5 \mathrm{~mm}$ with interquartile range of $4 \mathrm{~mm}$ over $2.1 \pm 1.9$ years. Endoscopic ultrasound done for morphologic change resulted in a change in diagnosis and management in $16 \%$ and $13 \%$ of cases, respectively.

Conclusion Endoscopic ultrasound has a modest but clinically significant role in impacting diagnosis and management for presumed mucinous cystic neoplasms when performed for the indication of cyst morphology change.

\section{Introduction}

Endoscopic ultrasound (EUS) is routinely used for patients undergoing surveillance for presumed cystic neoplasms (PCN), especially presumed mucinous cysts including mucinous cystic neoplasms (MCN) and intraductal papillary mucinous neoplasm
(IPMN). Although there has been a move away from routine EUS examinations for surveillance in favor of using noninvasive imaging with computed tomography (CT) or magnetic resonance imaging (MRI) instead, recent guidelines including the American Gastroenterological Association (AGA), [1] the Fukuoka, [2] more recently the revised Fukuoka, [3] and American Col- 
lege of Gastroenterology (ACG) [4] guidelines, published in 2015, 2012, 2017, and 2018, respectively, recommend EUS with fine-needle aspiration (FNA) for patients who on CT or MRI have a morphologic change (e.g. dilated main pancreas duct, cyst size $\geq 30 \mathrm{~mm}$, solid cystic component, and more recently increasing cyst growth rate in the revised Fukuoka guidelines). Although visualization and characterization of PCNs may be better achieved with EUS, the quality of existing noninvasive imaging such as CT or MRI scans may be sufficiently good that the true impact on diagnosis and management of EUS performed during surveillance of presumed mucinous cysts for the indication of new morphological change seen on noninvasive imaging remains unclear.

This matter is further complicated by interoperator variability in cyst characterization, measurement, and classification amongst different radiologists. One retrospective evaluation of PCNs (with pathologic confirmation) by two radiologists, using well-established morphologic features, agreed on correct diagnosis in only $60 \%$ of cases [5]. An additional study assessing interobserver variability in measurement of PCNs with MRI and the impact of measurement standards showed that the withinsubject standard deviation measurement variability only changed from $4.0 \mathrm{~mm}$ prior to standardization to $3.3 \mathrm{~mm}$ after introduction of measurement standards. In the same study, interobserver agreement, kappa, improved from 0.59 to 0.65 ( $P=$ $0.04)$, suggesting that there is significant and frequent interobserver variability in measurement of pancreatic cystic lesions with MRI, which could affect clinical management [6]. Additional studies have supported this real discrepancy in measurement of pancreatic cyst size between CT and MRI imaging, which becomes relevant in cases where imaging modality may be switched during follow-up either because of timing, availability, or patient characteristics which may preclude them from undergoing one particular type of study [7].

The primary aim of this study was to detect changes in diagnosis and/or management decisions based on addition of EUS results compared with noninvasive imaging alone. Furthermore, we aimed to determine which subgroup(s) of patients, if any, truly benefit from EUS when performed after a routine surveillance $C T$ or MRI shows a morphologic change.

\section{Patients and methods}

A single-institution pancreatic cyst database was queried for all patients who underwent esophagogastroduodenoscopy with EUS for evaluation of a PCN during surveillance after a noninvasive imaging study (CT or MRI) suggested a morphologic change such as cyst size increase, a cyst $\geq 30 \mathrm{~mm}$, change in main pancreatic duct (PD) caliber, or a cyst with a solid component during a defined period of time (January 1, 2013 - October, 2016).

Using available clinical and imaging data, diagnoses were classified both before and after EUS as: presumed mucinous without worrisome features (WF) or high-risk stigmata (HRS), with WF or with HRS, or non-mucinous lesions, using previously published guidelines [2]. Worrisome features were defined as clinical pancreatitis, cyst $\geq 3 \mathrm{~cm}$, thickened/enhancing cyst walls, main PD size $5 \mathrm{~mm}$ to $9 \mathrm{~mm}$, non-enhancing mural nodule, and/or abrupt change in caliber of pancreatic duct with distal pancreatic atrophy. High-risk stigmata were defined as obstructive jaundice in a patient with cystic lesion of the head of the pancreas, enhancing solid component within cyst, and/ or main PD $\geq 10 \mathrm{~mm}$. Non-mucinous lesions were categorized as either serous cystadenomas (SCA) or pseudocysts.

Presumed mucinous cysts were defined as unilocular or multifocal cysts with elevated carcinoembryonic antigen (CEA) levels (>192 ng/UL), positive mucinous cytology of any grade, communication of the cyst with the main PD, with lack of convincing evidence supporting a non-mucinous lesion such as a SCA or a pseudocyst. SCA were defined as microcystic lesions with or without a central calcification, as well as a low CEA ( $<2 \mathrm{ng} / \mathrm{UL})$ [8]. Pseudocysts were defined as lesions with pancreatic fluid collections arising in the setting of pancreatitis (without prior evidence of a pancreatic cyst), with or without confirmation of resolution of the pancreatic fluid collection with time or after drainage.

Management decisions were defined a priori as either surgical evaluation for all patients diagnosed with presumed mucinous lesions with HRS or for those with positive cytology or a mural nodule, or continued surveillance for all others. Both pre- and post-EUS diagnoses and management decisions were made separately in a blinded fashion to avoid bias.

We collected baseline characteristics such as size and location as reported by CT or MRI. Imaging reports reviewed included initial noninvasive imaging that first described presence of a PCN, the "baseline image"; the imaging that triggered the indication for EUS based on a change in pancreatic cyst morphology, the "trigger image"; and the noninvasive imaging report that immediately preceded the trigger image, the "comparison image." By comparing data gathered during these imaging studies, we extrapolated changes in morphologic characteristics including overall size of the cyst during surveillance period as well as the rate of change, "cyst change rate." We calculated and recorded "cyst change rate" by first calculating the overall growth, in millimeters, and then dividing by the number of months that had elapsed between imaging studies. Thus, "cyst change rate" represents the rate of change in size of the PCN. We also recorded any changes in caliber of the main PD. Furthermore, we evaluated the "cyst change rate" of each case that triggered the EUS in order to determine if there were factors which were predictive of a change in diagnosis or management.

Both diagnoses and management decisions were determined based on the clinical and radiologic imaging available at the time of the "trigger image" and again after the subsequent EUS was performed. Management decisions included continued surveillance with CT or MRI imaging with the same or increased or decreased frequency, or referring the patient for surgical evaluation.

Results are presented as mean \pm standard deviation or median with interquartile range when data are skewed. Categorical variables (e.g. presence of change in cyst size, cyst size $\geq 30 \mathrm{~mm}$, change in main PD caliber, presence of a solid cystic component) were analyzed using chi-square testing. A $P$ value 
$<0.05$ was considered statistically significant. Using logistic regressions analysis, we examined variables that could be used as predictors for a change in diagnosis or management postEUS compared to diagnosis and management pre-EUS. IRB approval was sought and obtained prior to the initiation of this study.

\section{Results}

Between January 2013 and October 2016, a total of 709 EUS examinations for evaluation of a PCN were completed at our institution. A total of 89 examinations were performed (M:F - 34:55, average $65.36 \pm 13$ years, range $15-82$ years) for the indication of cyst morphology change, including a change in cyst size ( 87 examinations), a cyst $\geq 30 \mathrm{~mm}$ (27 examinations), a change in PD caliber ( 8 examinations), and a cyst with a solid component (1 examination). Total surveillance time amongst these 89 cases was on average $3.58 \pm 2.57$ years.

\section{Presumed mucinous neoplasms}

A total of 70 pancreatic cyst EUS examinations were performed in 59 patients for presumed mucinous cyst of the pancreas (M: $\mathrm{F}-21: 38$, average $66.9 \pm 12$ years, range $28-82$ years) who had been under surveillance for $3.5 \pm 2.25$ years. Indications for EUS examinations included change in cyst size (68 examinations), a cyst $\geq 30 \mathrm{~mm}$ (10 examinations), a change in PD caliber (7 examinations), and a cyst with a solid component (1 examination). Preceding EUS, patients were imaged with CT or MRI at least twice, an earlier "comparison image" and a second, "trigger image", leading to the EUS. These two noninvasive imaging studies were done $2 \pm 1.9$ years apart during which time the lesion being surveyed grew $6.57 \pm 7 \mathrm{~mm}$ for a "cyst change rate " of $0.64 \pm 1 \mathrm{~mm}$. The time elapsed between the "trigger image" and the resulting EUS occurred at an average $3 \pm 4$ months after the "trigger image."

\section{Impact of EUS on diagnosis and management}

Prior to EUS, patients with presumed mucinous cysts of the pancreas were categorized as follows: without WF or HRS -52 cases (cyst size $18.6 \pm 6 \mathrm{~mm}$ ), with WF-17 cases (cyst size $28.8 \pm 18 \mathrm{~mm}$ ), and with HRS -1 case (cyst size, $22 \mathrm{~mm}$ ). Accordingly, the management plan was to continue surveillance with CT/MRI imaging in all cases with the exception of the one case with HRS, which would be referred for surgical evaluation.

Following EUS done for indication of morphologic change, the distribution of diagnosis changed ( $\triangleright$ Fig. 1 ). Of the 52 cases classified as presumed mucinous neoplasm without WF or HRS, 47 cases remained classified as the same after EUS. Of the remaining five cases, one case was changed to SCA, and four cases $(7 \%)$ were upgraded to a worse diagnosis - one neuroendocrine tumor (NET), one adenocarcinoma, one presumed mucinous neoplastic cyst with WF, and one presumed mucinous neoplastic cyst with high-grade dysplasia (HGD) considered to be malignant. Management decisions changed as well, with 3 cases (NET, adenocarcinoma, and HGD) all being referred for surgical evaluation (instead of ongoing surveillance) ( Fig. 2).

Of the 17 cases classified as presumed mucinous with WF prior to EUS, three were downgraded to presumed mucinous without WF, two were upgraded to a worse diagnosis - one malignancy and one presumed mucinous with HRS, and 12 remained presumed mucinous with WF of which nodules were detected in four cases ( $\mathbf{F i g . 3}$ ). Management decisions were impacted; six cases were referred for surgical evaluation (1 malignancy detected on FNA, 1 HRS, and 4 cases with nodules).

The single case classified as presumed mucinous with HRS prior to EUS was in fact detected to be a malignant lesion following EUS. Management did not change as the patient was referred for surgical evaluation regardless.

In total, EUS done for morphologic change resulted in a change in diagnosis in 11 cases (16\%), with 7 cases (64\%) being

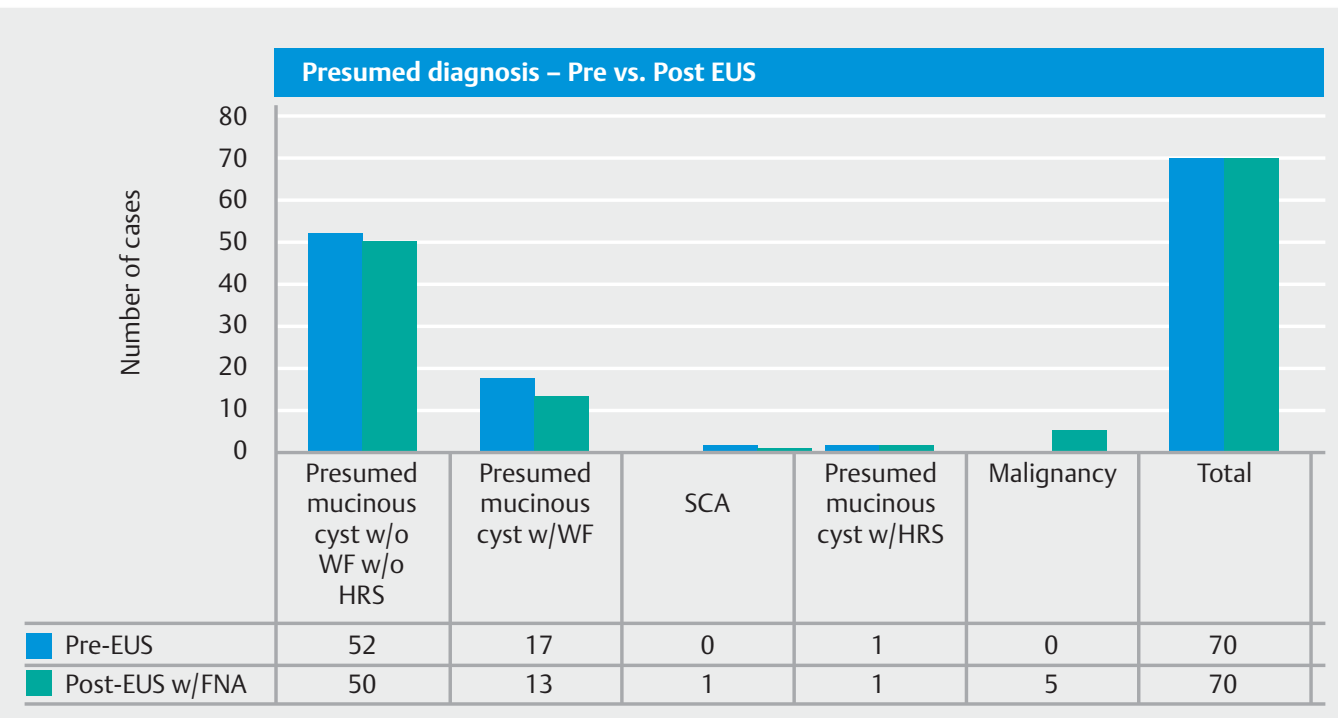

- Fig. 1 Changes in diagnosis in patients undergoing surveillance following EUS. 
a worse diagnosis. In addition, mural nodules were detected using EUS in four cases not detected on cross-sectional imaging, ranging from $2.4 \mathrm{~mm}$ to $11 \mathrm{~mm} \times 8 \mathrm{~mm}$ in size. In total, management decisions were changed in 9 cases (13\%) leading to surgical evaluation.

Using logistic regressions analysis, we examined variables that could be used as predictors for a change in diagnosis or management after EUS was done for morphologic change. Among our cohort, 47 cases demonstrated a "cyst change rate" $\geq 2 \mathrm{~mm} /$ year, of which 9 (19\%) had a change in diagnosis. This did not represent a significant correlation $(P=0.318)$. However, overall growth of $\geq 10 \mathrm{~mm}$ was found to be a significant predictor of change in diagnosis $P=0.01$, with 11 cases having $\geq 10 \mathrm{~mm}$ of growth and $5(45.5 \%)$ having a change to a worse diagnosis $(P=0.01)$. The indication of cyst size $\geq 30 \mathrm{~mm}$ and presence of any growth of a cyst, and presence of both features had significant correlation with changes in diagnosis $(P=0.015$, 0.004 , and 0.009 , respectively). ( $\triangleright$ Table 1$)$. There were no significant predictors of changes in management following EUS.

\section{Discussion}

The ability to accurately preoperatively classify pancreatic cysts in surveillance studies remains challenging, as no reliable method exists to consistently predict whether a PCN will degenerate to advanced malignancy. Identifying high- and low-risk lesions reliably could lead to early referral and intervention in patients whose lesions would eventually progress to malignancy and allow for periodic imaging in those cases where progression to malignancy is unlikely (but still possible) [9]. For example, whereas the rates of progression to malignancy for SCA are negligible, those for IPMNs carry a wide range of malignant potential $(5 \%-62.2 \%)[2,10,11]$.

Several studies report poor accuracy rates for classifying pancreatic cysts preoperatively, however, most of these are either out of date or focused on the inaccuracies of separating main duct from branch duct IPMN [5, 12]. With increasing interest in pancreatic cyst surveillance, there are growing guidelines for more accurate preoperative diagnoses and management of pancreatic cysts, however, they may be understating the modest but significant role of EUS [1,2].

This becomes problematic for practitioners who are deciding between referring patients for aggressive surgical intervention or conservative surveillance. Patients who undergo aggres-

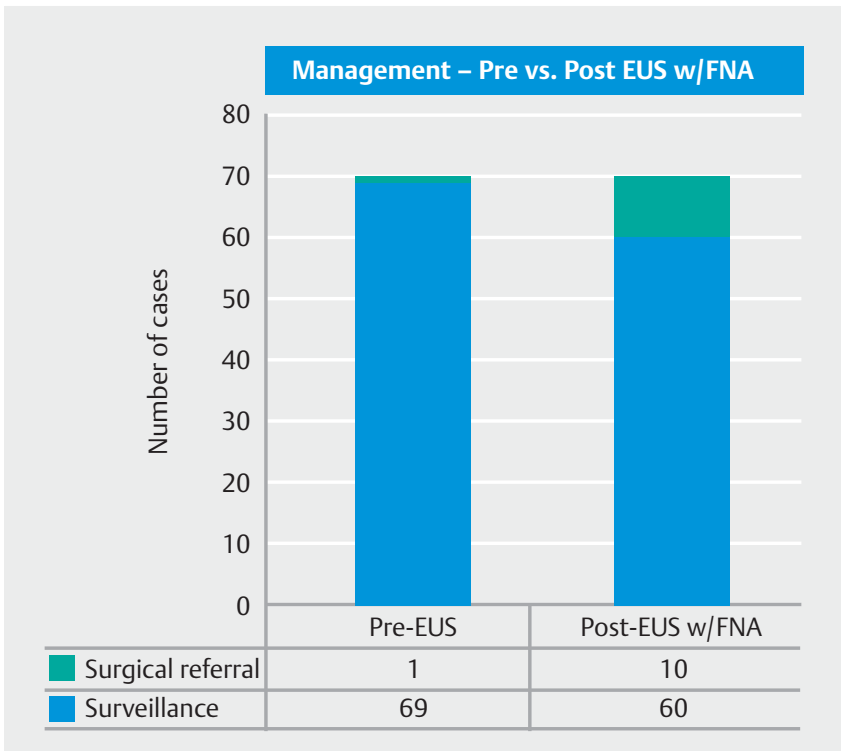

Fig. 2 Changes in management in patients undergoing surveillance following EUS.

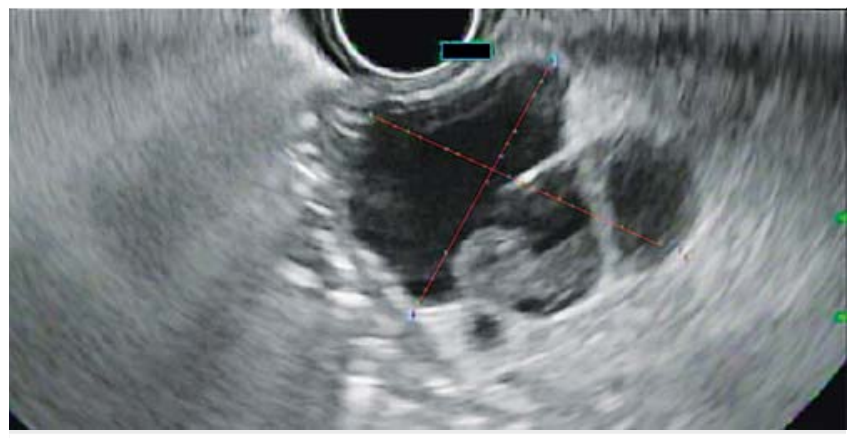

- Fig. 3 Mural nodule detected on EUS not detected on prior cross-sectional imaging.

sive pancreatic surgical intervention, according to a longitudinal study including 851 patients who underwent resection for a cystic neoplasm of the pancreas, are at considerable risk for postoperative complications and mortality [13]. These risks of malignant degeneration must be balanced with risks and benefits associated with definitive surgical management, patient comorbidities, and life expectancy. Recent guidelines have fa-

- Table 1 Changes in diagnosis pre- vs. post-EUS.

\begin{tabular}{|l|c|c|c|}
\hline & Total \# of EUS procedures & Change in Diagnosis post EUS/FNA & $P$ value \\
\hline Indication & & & 0 \\
\hline Cyst Growth & 68 & 10 & 0.004 \\
\hline Cyst $\geq 30 \mathrm{~mm}$ & 10 & 3 & 0.015 \\
\hline Growth + Cyst $\geq 30 \mathrm{~mm}$ & 10 & 3 & 0.009 \\
\hline Cyst Growth $\geq 10 \mathrm{~mm}$ & 11 & 5 & 0.01 \\
\hline Cyst Change rate $\geq 2 \mathrm{~mm} /$ year & 47 & 9 & 0.318 \\
\hline
\end{tabular}


vored less invasive imaging both during initial evaluation and management during surveillance, as well as even making recommendations about stopping surveillance in certain subgroups (e.g. those without positive features on cross-sectional imaging over 5-year surveillance and those who lack positive cytology and positive features on initial EUS followed by stable cross-sectional imaging during 5-year surveillance) [1].

Our data suggest that use of selective EUS impacts both diagnosis and management of pancreas cystic neoplasms during surveillance. Overall, EUS performed during routine surveillance of presumed mucinous neoplasms done for a change in morphology resulted in a change in diagnosis and management in $11(16 \%)$ and 9 cases (13\%), respectively. This is not surprising based on existing data on the impact of EUS on diagnosis and management [14-17]. When comparing noninvasive cross-sectional imaging with EUS in terms of diagnostic accuracy, one retrospective review noted that diagnostic accuracy for neoplastic cysts (defined as cystic pancreatic ductal adenocarcinoma, cystic pancreatic neuroendocrine tumor, MCN, IPMN, and solid pseudopapillary neoplasm) improved by $36 \%$ with the addition of EUS compared to CT or MRI alone [18]. When noninvasive imaging alone is used to make preoperative diagnosis in patients undergoing surgical intervention, accurate diagnosis are made in $30 \%$ and $29 \%$ of cases of mucinous cystadenoma and mucinous cystadenocarcinoma as demonstrated in a large multicenter study [5]. This is due in large part to the superior imaging with EUS and its ability to detect cystic features such as nodules ( $\mathbf{F i g . 4 a , ~} \triangleright$ Fig.4b, and $\triangleright$ Fig.4c). Among our cohort of patients, 4 (6\%) were found to have a nodule using EUS not detected with cross-sectional imaging. It has been suggested that significant proportion of echogenic nodules identified on EUS are in fact mucus, however, experienced endosonographers are able to perform maneuvers during EUS examinations, and thereby increase diagnostic sensitivity and specificity. These maneuvers include changing patient body position and attempting to dislodge echogenic materials from needle tip prior to performing fine-needle aspiration (FNA) [19]. Among mural nodules detected in our series, all would have qualified for surgical evaluation per Fukuoka guideline, as presence of mural nodule regardless of size represents indication for surgical evaluation. Newer European guidelines include a size cut-off of $>5 \mathrm{~mm}$, above which surgical evaluation is absolutely indicated [20]. Adding FNA resulted in a change in diagnosis in an additional 5 cases (7\%) due to the added benefit of cytology and tumor marker data, making supplementation of noninvasive imaging with EUS examinations valuable in evaluation of patients with cystic lesions. In a prospective study looking at 302 patients with pancreatic cysts including 110 measuring $<30 \mathrm{~mm}$, the overall impact of EUS on management was calculated to be $72 \%$ of cases [21]. In our cohort, diagnosis was upgraded to a worse diagnosis or the same diagnosis with higher-risk features in 11 patients (16\%), with 9 (13\%) additional patients necessitating surgical evaluation.

Overall growth rate of cystic neoplasms, and not just the actual size should also be taken in to consideration when making management decisions. It has been suggested that a growth rate of $\geq 2 \mathrm{~mm} /$ year and total growth $\geq 10 \mathrm{~mm}$ are predictive of
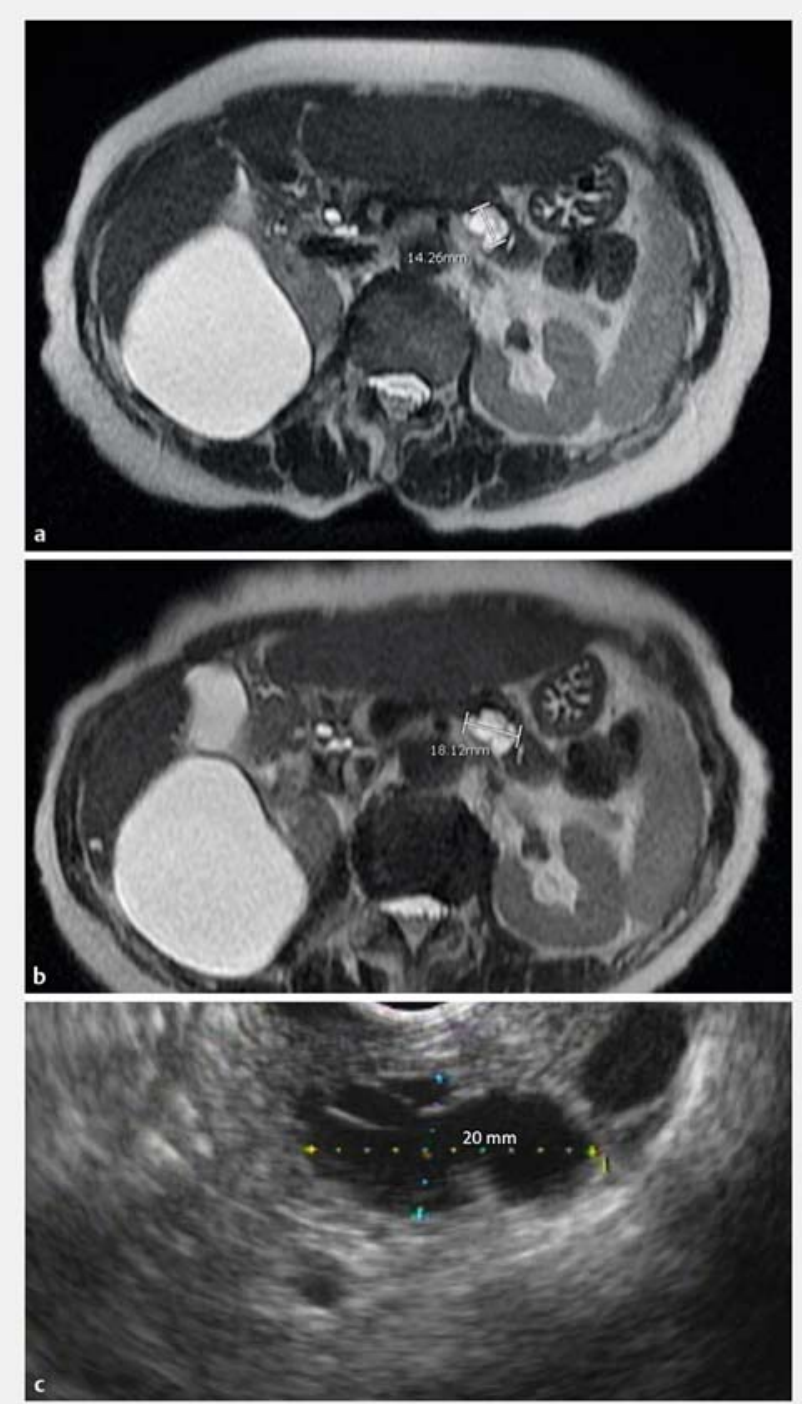

Fig. 4 Image series demonstrating a the same pancreas cyst as seen on initial cross-sectional imaging, b follow-up trigger crosssectional imaging, and c subsequent EUS imaging.

BD-IPMN malignant degeneration and should be considered worrisome features [20, 22, 23]. In fact the most recent revision of the Fukuoka guidelines does include a rate of cyst size change of $>5 \mathrm{~mm}$ within 2 years as a worrisome feature necessitating EUS evaluation for further evaluation [3]. This conclusion was drawn from following a cohort of 284 patients with presumed BD-IPMN without WF or HRS for an average of 56 months [22]. On closer examination of the 9 patients who developed malignant lesions amongst this cohort, it was found that their growth rate was $18.6 \mathrm{~mm} /$ year compared with 0.8 $\mathrm{mm} /$ year in patients who did not develop malignant lesions. In our cohort of patients with presumed mucinous cysts, the average pancreatic cyst growth rate was $7.68 \mathrm{~mm} /$ year. Among patients whose post-EUS diagnosis was worse than pre-EUS, a pancreatic cyst growth rate of $12.48 \mathrm{~mm} /$ year was measured and was higher still in patients who developed malignant lesions, $14.58 \mathrm{~mm} /$ year, compared with $5.76 \mathrm{~mm} /$ year in patients 
with no change in diagnosis. From our cohort, the pancreatic cyst growth rate of $\geq 2 \mathrm{~mm} /$ year could be used as a predictor for a worse diagnosis following EUS, however, the sample size may not be large enough to draw a significant conclusion $(P=$ $0.318)$. Similarly, considering overall growth in cyst size with various cutoffs can also serve as a reliable predictor for changes in diagnosis, thereby supporting use of EUS in patients who demonstrate significant growth during surveillance. In our cohort, patients with presumed mucinous neoplasms of the pancreas that grew by $\geq 10 \mathrm{~mm}$, regardless of the rate of growth, were more likely to have a change in diagnosis following EUS ( $P=$ $0.01)$.

There are several limitations of this study. A limitation of surveillance studies in general, including our own, is that the majority of pancreatic cysts are not operated on and do not have a definitive diagnosis. By virtue of limitation in diagnostic accuracy of both imaging and cyst fluid biomarkers, diagnoses remain presumed based on available imaging and clinical data. In addition, our study is retrospective and thus is open to biases in terms of defining diagnosis and management decisions. To overcome these inherent biases, both diagnoses and management decisions were defined a priori and were applied to both the pre- and post-EUS scenarios in a blinded fashion. In addition, relying on imaging reports to document changes in cyst size is concerning due to the lack of standardization of measurement. CT or MRI, rather than EUS, are appropriate for surveillance in many cases of PCNs, especially those $\leq 30 \mathrm{~mm}$, without thickened or enhancing walls, main PD size $<5 \mathrm{~mm}$, non-enhancing nodule, and without abrupt change in main PD caliber [2]. However, the problem arises when considering cases where these characterizations cannot be reliably made and the fact that interobserver agreement in classifying pancreatic cysts using MRI is moderate at best, with variability in cyst size characterization amongst radiologists measured to be $4 \mathrm{~mm}$, and agreement in characterization in just $51 \%$ of cases $[6,24]$. Discrepancies are also described between radiologists assessing patients for pancreatic cystic neoplasms on CT compared to MRI, with a median difference in cyst measurement of $1.5 \mathrm{~mm}$ between the two modalities, which led to an inappropriate change in surveillance regimen in $6 \%$ of cases [7]. EUS, like CT and MRI, is also prone to interobserver discrepancy in interpretation $[25,26]$. It is to be noted that our evaluation and recording of presumed mucinous lesions on noninvasive imaging did not include reevaluation of actual films. While reviewing films would allow us to ensure consistent and accurate measurements of presumed mucinous lesions, it is unlikely that this is what actually happens consistently in clinical practice. This draws attention to the importance of consistent and standardized techniques for measuring and reporting presumed mucinous lesions by radiologists. However, use of the imaging reports rather than formally documenting and remeasuring all the imaging reflects the "real-world" scenario of how patients with pancreatic cysts are managed. Prospectively, standardization of how pancreatic cysts are documented and reported, in addition to formal multidisciplinary imaging review, will improve this issue. Furthermore, our study was also limited by the fact that CEA measurements were not available in several patients.

Currently pancreatic cyst fluid biomarkers (e.g. CEA, Kras, GNAS) may have a role in differentiating mucinous from nonmucinous cysts, however, not with sufficient accuracy to ignore other clinical and radiologic data when making decisions regarding surgical or surveillance management [27 - 30]. Pancreatic cyst fluid biomarkers are currently inaccurate for making the diagnosis of advanced malignancy including HGD and cancer [31]. However, it is possible that with development of nextgeneration DNA sequencing markers and methylation markers, this may improve. It is also therefore possible that in the future these newer cyst fluid biomarkers may be incorporated into clinical guidelines about surveillance and surgical management.

Finally, although this study focused on the impact of EUS on diagnosis and/or management, we were unable to calculate the impact of EUS on overall outcome in this patient subgroup. A future direction of our research will be to inquire as to the final outcomes of patients who were referred for surgical evaluation (if an intervention was undertaken that changed their overall outcome), as there are very few studies looking at the role of EUS in overall outcome in patients with pancreatic cysts [32].

\section{Conclusion}

In evaluating the role of EUS and whether there is added benefit in diagnosis and management of patients who are enrolled in surveillance programs for presumed mucinous lesions, EUS carries a modest but clinically significant role in changing diagnosis and management when performed for the indication of morphologic change. Furthermore, predictors such as interval growth between noninvasive imaging studies as well as the rate of growth may also be valuable clinical predictors of worsening clinical diagnosis after EUS.

\section{Competing interests}

None

References

[1] Vege SS, Ziring B, Jain R et al. American gastroenterological association institute guideline on the diagnosis and management of asymptomatic neoplastic pancreatic cysts. Gastroenterology 2015; 148 : 819-822; quiz e812-813

[2] Tanaka M, Fernandez-del Castillo C, Adsay V et al. International consensus guidelines 2012 for the management of IPMN and MCN of the pancreas. Pancreatology 2012; 12: 183-197

[3] Tanaka M, Fernandez-Del Castillo C, Kamisawa T et al. Revisions of international consensus Fukuoka guidelines for the management of IPMN of the pancreas. Pancreatology: official journal of the International Association of Pancreatology 2017; 17: 738 - 753

[4] Elta GH, Enestvedt BK, Sauer BG et al. ACG Clinical Guideline: Diagnosis and Management of Pancreatic Cysts. Am J Gastroenterol 2018; 113: $464-479$ 
[5] Procacci C, Biasiutti C, Carbognin G et al. Characterization of cystic tumors of the pancreas: CT accuracy. J Comput Assist Tomogr 1999; 23: $906-912$

[6] Dunn DP, Brook OR, Brook A et al. Measurement of pancreatic cystic lesions on magnetic resonance imaging: efficacy of standards in reducing inter-observer variability. Abdom Radiol (NY) 2016; 41: $500-$ 507

[7] Boos ], Brook A, Chingkoe CM et al. MDCT vs. MRI for incidental pancreatic cysts: measurement variability and impact on clinical management. Abdom Radiol (NY) 2017; 42: 521 - 530

[8] Tseng JF, Warshaw AL, Sahani DV et al. Serous cystadenoma of the pancreas: tumor growth rates and recommendations for treatment. Ann Surg 2005; 242: 413-419; discussion 419-421

[9] Kolb JM, Argiriadi P, Lee K et al. Higher growth rate of branch duct intraductal papillary mucinous neoplasms associates with worrisome features. Clin Gastroenterol Hepatol 2018; 16: 1481- 1487

[10] Crippa S, Salvia R, Warshaw AL et al. Mucinous cystic neoplasm of the pancreas is not an aggressive entity: lessons from 163 resected patients. Ann Surg 2008; 247: 571 - 579

[11] Galanis C, Zamani A, Cameron JL et al. Resected serous cystic neoplasms of the pancreas: a review of 158 patients with recommendations for treatment. J Gastrointest Surg 2007; 11: $820-826$

[12] Curry CA, Eng J, Horton KM et al. CT of primary cystic pancreatic neoplasms: can CT be used for patient triage and treatment? AJR Am J Roentgenol 2000; 175: 99-103

[13] Valsangkar NP, Morales-Oyarvide V, Thayer SP et al. 851 resected cystic tumors of the pancreas: a 33-year experience at the Massachusetts General Hospital. Surgery 2012; 152: S4-12

[14] Ahmad NA, Kochman ML, Lewis JD et al. Can EUS alone differentiate between malignant and benign cystic lesions of the pancreas? Am J Gastroenterol 2001; 96: 3295-3300

[15] Canto MI, Hruban RH, Fishman EK et al. Frequent detection of pancreatic lesions in asymptomatic high-risk individuals. Gastroenterology 2012; 142: $796-804$; quiz e714-795

[16] Koito K, Namieno T, Nagakawa T et al. Solitary cystic tumor of the pancreas: EUS-pathologic correlation. Gastrointest Endosc 1997; 45: $268-276$

[17] Lu X, Zhang S, Ma C et al. The diagnostic value of EUS in pancreatic cystic neoplasms compared with CT and MRI. Endosc Ultrasound 2015; 4: 324-329

[18] Khashab MA, Kim K, Lennon AM et al. Should we do EUS/FNA on patients with pancreatic cysts? The incremental diagnostic yield of EUS over CT/MRI for prediction of cystic neoplasms Pancreas 2013; 42: $717-721$
[19] Zhong N, Zhang L, Takahashi N et al. Histologic and imaging features of mural nodules in mucinous pancreatic cysts. Clin Gastroenterol Hepatol 2012; 10: 192 - 198, 198 e191-192

[20] European Study Group on Cystic Tumours of the Pancreas. European evidence-based guidelines on pancreatic cystic neoplasms. Gut 2018; 67: $789-804$

[21] Ardengh JC, Lopes CV, de Lima-Filho ER et al. Impact of endoscopic ultrasound-guided fine-needle aspiration on incidental pancreatic cysts. A prospective study. Scand J Gastroenterol 2014; 49: 114-120

[22] Kwong WT, Lawson RD, Hunt G et al. Rapid growth rates of suspected pancreatic cyst branch duct intraductal papillary mucinous neoplasms predict malignancy. Dig Dis Sci 2015; 60: 2800-2806

[23] Kang M], Jang JY, Kim SJ et al. Cyst growth rate predicts malignancy in patients with branch duct intraductal papillary mucinous neoplasms. Clin Gastroenterol Hepatol 2011; 9: 87-93

[24] de Jong K, Nio CY, Mearadji B et al. Disappointing interobserver agreement among radiologists for a classifying diagnosis of pancreatic cysts using magnetic resonance imaging. Pancreas 2012; 41: $278-282$

[25] Ahmad NA, Kochman ML, Brensinger C et al. Interobserver agreement among endosonographers for the diagnosis of neoplastic versus nonneoplastic pancreatic cystic lesions. Gastrointest Endosc 2003; 58: $59-64$

[26] Oh HC, Kim MH, Hwang CY et al. Cystic lesions of the pancreas: challenging issues in clinical practice. Am J Gastroenterol 2008; 103: 229-239; quiz 228, 240

[27] Farrell J]. Prevalence, Diagnosis and Management of Pancreatic Cystic Neoplasms: Current Status and Future Directions. Gut Liver 2015; 9: $571-589$

[28] Singhi AD, Nikiforova MN, Fasanella KE et al. Preoperative GNAS and KRAS testing in the diagnosis of pancreatic mucinous cysts. Clin Cancer Res 2014; 20: 4381 - 4389

[29] Springer S, Wang Y, Dal Molin M et al. A combination of molecular markers and clinical features improve the classification of pancreatic cysts. Gastroenterology 2015; 149: $1501-1510$

[30] Wu J, Matthaei H, Maitra A et al. Recurrent GNAS mutations define an unexpected pathway for pancreatic cyst development. Sci Transl Med 2011; 3: 92 ra66

[31] Sreenarasimhaiah J, Lara LF, Jazrawi SF et al. A comparative analysis of pancreas cyst fluid CEA and histology with DNA mutational analysis in the detection of mucin producing or malignant cysts. JOP 2009; 10 : $163-168$

[32] Al-Haddad M, Hajj II El, Eloubeidi MA. Endoscopic ultrasound for the evaluation of cystic lesions of the pancreas. JOP 2010; 11: 299-309 